duction of quartz fibres, accompanied by a demonstration. Their physical properties, and their recent use in optics for the rapid transmission of images, were discussed. H. M. Appleyard described how the causes of faults in textiles, many of which are not obvious until the fabric has been dyed and finished, can be ascertained by microscopical examination. Faults may be due to adulterant fibre in the blend or to solid matter attached to the fibres, such as pieces of skin. Identification tests include normal histological techniques or microchemical tests followed by examination under the conventional or phase-contrast microscope. The systematic study of the removal, from a variety of textile fibres, of compounds of the same type as those found in fatty dirt from worn garments was described by R. E. Wagg. He discussed the action of several different classes of detergent, builders and other additives.

The measurement of the diameter of glass fibres, either mechanically drawn or steam-blown fibres, can be carried out by means of a projection microscope, and A. Pimblett described this method, together with the techniques required to prepare mounts of crosssections of fibres. By this method of viewing, variations in diameter and cross-sectional shape, and the bonding together of steam-blown fibres to form insulation products, are revealed. The appearance and handle of acetate fabrics are profoundly affected by the cross-sectional shape and surface of the filament. These characteristics require to remain constant in a given spinning run, and $\mathrm{L}$. Krause described how, as a mothod of control, yarns are examined microscopically for lustre and crosssectional shape.

At the exhibition held in conjunction with the symposium, D. E. Bradley showed electron-micrographs of various fibres examined under the reflexion microscope by means of replicas. Faults causing electrical failure in thin oriented polystyrene-film capacitors have been identified microscopically as particles of dandruff introduced by the machine operators, and H. F. Church exhibited several of these faults, together with photomicrographs illustrating their structure. Dr. M. Dempsey and Miss B. M. Haines demonstrated a melting-point apparatus, adapted for measuring microscopically the shrinkage temperature of isolated leather fibres, and H. W. Emerton, J. Watts and D. H. Page showed photo- and electron-micrographs of various papermaking fibres.

The penetration and distribution of stain in a damaged rayon fibre can determine the type of damage, and Dr. D. G. Drummond and J. E. Ford exhibited photomicrographs illustrating the different types of fibre damage. Electron-micrographs of the surface of textile fibres made by the replica technique in the transmission electron microscope were also shown. Photographs of apparatus and of Fizeau fringes around points of contact between fibres and a quartz plate were shown by D. B. Gowers, while C. F. Griffiths exhibited specimens and photomicrographs of glass fibres containing crystalline inclusions; the inclusions may originate from the raw materials, or the refractories of the furnace or devitrification of the glass itself, and they can be identified by means of the polarizing microscope or by the X-ray diffraction method.

Photomicrographs illustrating the differences between textile fibres of vegetable origin were contributed by C. J. Jarman; from the size of the cell and the number of cells to a fibre it is possible to identify the fibre. Photo- and electron-micrographs of the fibres in human connective tissues were shown by Dr. K. Little, and J. A. Mason displayed samples of 'Perspex' and polythene that had been used for insulations and were exhibiting erosion and breakdown in the form of dendritic channels formed by local stress and heating by electrical discharges. T. S. Millen and R. S. Page demonstrated the use of the electron microscope for the examination of the surface of fibres by the reflexion method.

Slides and photomicrographs were used by $A$. Pimblett for demonstrating the method of measuring the diameter of glass fibres, and Dr. F. Stern showed photomicrographs and X-ray diffraction patterns of coir fibre, demonstrating the special fibrillar orientation of the cellulose microfibrils. Quartz fibres and photographs illustrating the methods for producing the fibres were exhibited by Dr. A. E. J. Vickers, and R. E. Wagg and Miss Gayner Stephens showed by means of photomicrographs the effect of various detergents on fatty dirt contaminating textile fibres. C. R. Watts contributed photomicrographs illustrating various yarns and fabrics, including modern developments in bonded and coated fibres; some common faults and their causes were also shown.

Electron micrographs and stereomicrographs showing the surface characteristics and microfibrillar texture of keratin fibres were exhibited by Mr. H. J. Woods and Dr. J. Sikorski. Miss E. Frei and Prof. R. D. Preston exhibited longitudinal and transverse sections of coniferous woods $300 \mathrm{~A}$. thick for study in the electron microscope; the electron-micrographs showed the fine spiral structure of the various layers of the wall.

\section{RHEOLOGY OF PROTEINS}

A SYMPOSIUM on the "Rheology of Proteins" was held in London on January 20 by the British Society of Rheology. Four papers were presented, each of which aroused considerable discussion. The chairman, Mr. A. G. Ward (British Gelatine and Glue Research Association), in his opening remarks, outlined the wide scope covered by the title of the symposium. Proteins, the mechanical properties of which play a vital part in their function. ing in living organisms, include serum proteins, epidermal and connective tissue proteins, muscle proteins, many cellular proteins, mucoproteins and mucopolysaccharides, etc. The rheological properties of certain of these also contribute to their subsequent use in meat, egg and other protein foods, in protein fibres, in gelatin and animal glue, and in other products. In the background, for all these substances, rest the two common problems of the covalently bonded structure of the protein molecules and the secondary forces-ionic, hydrogen bonding, and van der Waals interactions-which link the molecules together to form macroscopic aggregates. Only by isolating the simplest systems will it be possible to solve the many complex problems.

Dr. E. J. Ambrose (Chester Beatty Research Institute), in his paper on the formation of structure in protein solutions, was concerned with the conditions in which a two-phase system can be formed, the protein or related component being organized or ordered in the concentrated phase. The properties of a macromolecule determining its tendency to form such structures are particle size and flexibility, the 
latter influencing the disturbance by Brownian movement, shape, the interaction forces, and the degree of reversibility of the changes. If the interaction forces are strong, there is a greater tendency to a disordered separation of the second phase, whereas weak forces suffice to make the necessary links only when a degree of order is attained. These considerations were illustrated by the structures obtainable with poly- $\gamma$ benzyl-L-glutamate in chloroform solution, and with insulin. Birefringent structures show orientation to be occurring when phase separation occurs.

Primary interest is on the behaviour of the cell nucleus and its component nucleo-proteins. Whereas nucleic acid alone fails to give phase separation, nucleoprotein forms fibrous structures, showing birefringence and dichroism. The existence of different structures at different concentrations for nucleo-proteins is shown by a discontinuity in the relation between concentration and diffusion constants. No discontinuity is observed for the nucleic acid alone. The techniques and theories have been applied to the structure of chromosomes and also to the formation of the tail of the sperm during meiotic division.

In the second paper, Dr. A. Wassermann (University College, London) described his work on a viscosimetric investigation into the influence of $p \mathrm{H}$ and ionic strength on molecular shape of a structural muscle protein. Two views have been put forward concerning the actomyosin molecule, the first regarding it as a stiff rod or elongated ellipsoid of revolution and the second treating it as a flexible random coil. Use of the mixed-bed ion-exchange resin column enables actomyosin solution of very low salt content to b) prepared. Alkali additions give solutions, the 'intrinsic viscosities' of which extrapolated to zero rate of shear, and to zero concentration, are a maximum in the region of $p \mathrm{H} \mathrm{8-9.} \mathrm{At} \mathrm{higher} p \mathrm{H}$, lower values are obtained. No peak is observed on the acid side. The absence of change below $p \mathrm{H} 4$ is assumed to arise from anion binding neutralizing the charges developed. The peak is considered to arise from the stretching out of the molecule by the repulsion of negative charges present at the $p \mathrm{H}$ 's concerned. Beyond a certain $p \mathrm{H}$ the concentration of counter ions becomes sufficient to reduce the forces between the charges, so that a reduction in intrinsic viscosity' occurs; similarly, the addition of neutral salt in sufficient amount reduces the changes in intrinsic viscosity to zero. All these observations are fully compatible with the random coil hypothesis but are inconsistent with the stiff rod theory.

In the third paper at the symposium, on some rubber-like and relaxation properties of gelatin, $\mathbf{M r}$. D. W. Jopling (Kodak, Ltd.) demonstrated that gelatin films maintained at a water content of 25-30 per cent by immorsion in $85 / 15$ alcohol/water or by exposure to high humidity show rubber-like extensibilities of up to several hundred per cent. This is best shown when the ordered arrangement, brought about by drying, has been destroyed by immersion in boiling methanol.

By cross-linking gelatin molecules with formaldehyde, measurements on the resultant film can be carried out at temperatures at which normal gelling forces are absent, for example, $60^{\circ} \mathrm{C}$. Stress relaxation then follows the Maxwell relation $\log$ (stress) $=$ $A-B$ (time), whereas at room temperature stress is proportional to $\log (\operatorname{tim} \theta)$. It is assumed that at $25^{\circ} \mathrm{C}$. the mechanism is primarily the release of secondary bonds, with a wide spectrum of relaxation times. whereas at $60^{\circ} \mathrm{C}$. only primary bonds are broken. At $40^{\circ} \mathrm{C}$. both processes can be observed to occur in succession. The form of the extension-tensile stress relation for gelatin cross-linked with formaldehyde at $60^{\circ} \mathrm{C}$. follows the theoretical curve for an ideal rubber-like system up to extension ratios of $4: 1$. The values for the chain molecular weight between cross-links deduced from the theory appear high; the reasons for this are not clear.

The final paper, by Dr. J. Brooks and Mr. H. P. Hale (Low Temperature Research Station), on the mechanical properties of the ovomucin gel of the egg, was given by Dr. Brooks. The thick white fraction of the hen's egg consists of a gel in which a transparent phase is separated by a series of parallel bands of microscopic fibres. After the egg is laid, the volume of thick white decreases at a rate depending on the temperature. The mechanical properties of the gel have been investigated by a method originally devised by Freundlich, in which the displacement of a minute nickel particle inserted in the gel is measured microscopically. The force is applied by means of a magnetic field. For stresses of short duration, the displacements are elastic in character in both the transparent phase and the bands. The bands are anisotropic. The relative values of the rigidities are converted into absolute units by means of a series of measurements on gelatin gels. Measurements have been made on fresh eggs and on eggs stored at $25^{\circ}$ and $0^{\circ} \mathrm{C}$., and it has been found that there is a close relation between the rigidity of the thick white fraction and its volume, irrespective of the reason for the volume difference.

A. G. WARD

\section{SOME RECENT DEVELOPMENTS IN MAGNETISM}

$\mathrm{T}$ HE Electronics Group of the Institute of Physics held an informal symposium in London during September 23-24 on "Some Recent Developments in Magnetism", at which seven papers were read and discussed. The first speaker, Dr. E. W. Gorter (Philips Laboratories, Eindhoven), outlined the theory of the magnetic properties of the ferrite group of materials. They have the spinel type of structure, in which the metal ions occupy two different types of site, and the interactions between the ions are such that the total magnetic moment is roughly equal to the difference between those for the ions in the two types of site. Dr. Gorter showed why this relation might not be exact, and how various anomalous shapes of saturation/temperature curve can occur; he demonstrated a striking example, namely, a material the spontaneous magnetization of which is reversed as the temperature rises. The properties of ferrites can be used to infer the relative strengths of the interactions between ions in various configurations, and the evidence so obtained supports the theory of super-exchange.

Ferrites are insulators, some of them having resistivities of the order of $10^{5} \mathrm{ohms}$, and can therefore be studied in fields alternating even at microwave frequencies, whereas any similar experiments with metals are largely prevented by the effects of eddy currents. J. Watkins (G.E.C. Research Laboratories, Wembley) described the Faraday rotation and the transverse phase-shift, two effects observed in such experiments. The latter effect is a variation 